\title{
PARAMETRIC ANALYSIS OF SHEAR PANEL DAMPERS UNDER HIGH AXIAL COMPRESSION
}

\author{
Zhiyi Chen ${ }^{1,2, *}$, Hui Fan ${ }^{3}$ and Guoqiang Bian ${ }^{3}$ \\ ${ }^{1}$ Associate Professor, Department of Geotechnical Engineering, Tongji University, Shanghai 200092, China \\ ${ }^{2}$ Key Laboratory of Geotechnical and Underground Engineering of \\ Ministry of Education, Shanghai 200092, China \\ ${ }^{3}$ Department of Geotechnical Engineering, Tongji University, Shanghai 200092, China \\ *(Corresponding author: E-mail: zhiyichen@tongji.edu.cn)
}

Received: 21 December 2012; Revised: 18 March 2013; Accepted: 27 December 2013

\begin{abstract}
To explore the hysteretic behaviors of shear panel dampers under axial compression, numerical analyses were carried out using the general-purpose finite element software $A B A Q U S$. Two important parameters, namely the axial compression ratio and the web slenderness parameter, were selected as governing parameters. To trace the material nonlinearity, a modified two-surface model was adopted to simulate the constitutive relations of the steel material under cyclic shear loading. Discussions focused on the hysteretic behavior, maximum shear strength, and energy dissipation performance concerning the axial compression ratio. It was found that axial compression deteriorates the hysteretic behavior and energy dissipation performance of shear panel dampers. In addition, under high axial compression, a small web slenderness parameter leads to unexpected brittle failure.
\end{abstract}

Keywords: Shear panel damper, High axial compression ratio, Hysteretic energy dissipation, Underground structure, Numerical simulation

\section{INTRODUCTION}

Evidence from recent seismic disasters, especially the 1995 Kobe earthquake in Japan, indicates that underground structures are damaged seriously during a severe earthquake. Extensive studies and applications [1-4] have focused on shear panel dampers (SPDs) incorporated into ground buildings and bridges, which serve as a type of passive structural control device to dissipate seismic input energy. SPDs are able to dissipate a large amount of energy through plastic yielding and hysteretic deformation of the metal material. Characterized by simple configuration, and convenient replacement and maintenance, SPDs are used widely in ground structures and show outstanding energy dissipation performance and seismic control properties. However, both the application and research of SPDs in underground structures are still in their infancy [5].

In contrast with the design of ground structures, the seismic design of underground structures must consider fully the adverse effects of high axial compression on SPDs. This is because the columns of underground structures not only bear loading transferred from an upper structure [6], but they also suffer very strong dynamic axial forces due to the vertical vibration of overburdened soil under the effect of vertical ground motion [7]. Real failure modes and results of numerical analysis [8,9] have shown that the collapse of columns at the Dakai subway station was caused primarily by the joint action of the horizontal seismic shear force and superior axial compression.

To date, research has concentrated on the hysteretic behavior of SPDs, such as the effects of initial deformation, buckling and post-buckling phenomena, ultimate shear bearing capacity, and energy dissipation capacity. In research on axial compression, Sasaki and Tanaka [10] explored the effect of static axial compression on the hysteresis curves of SPDs fabricated of low-yield-point steel. They also empirically obtained an allowable level of axial compression. Experiments on two-way stiffened SPDs made of low-yield-point steel were carried out by Nakashima et al. [11]. Results showed that when the axial compression ratio reached 0.3, hysteresis curves were pinched 
conspicuously, but that the ability of the SPDs to dissipate energy was not reduced significantly because of the strain hardening of the steel material.

There has been relatively little research on the hysteretic behavior of SPDs under high axial compression loading. Chen et al. [5] conducted cyclic shear tests on five SPDs fabricated of Q235B mild steel. According to the code of Steel Plates for Building Structure [12], the yield stress and maximum stress of Q235B steel are about $235 \mathrm{MPa}$ and $400-510 \mathrm{MPa}$, respectively. Elongation is equal to or more than $23 \%$. These SPDs were designed to be capable of sustaining high axial compression induced in an underground structure by an earthquake. The experimental results revealed that shear capacity and ductility decrease with an increase of the axial compression ratio. When the axial compression ratio was 0.7 , the cumulative energy consumption of the SPDs was almost half that for the case of a ratio of 0.3. In the present study, a comprehensive parametric study is carried out through numerical simulation to overcome the shortcomings of indoor tests, for which the number of specimens is rather limited and duplication is difficult and expensive. The validity of the numerical model and the accuracy of the simulation results are initially verified by indoor test results. Then, the axial compression ratio and the shear-web slenderness are taken as the two most significant control parameters. Hysteretic behavior, maximum shear strength, and energy dissipation performance are discussed concerning a high axial compression ratio.

\section{NUMERICAL MODEL}

\subsection{Main Parameters of SPDs}

Figure 1 is a schematic diagram of an SPD. Generally, the shear web, which comprises the primary energy dissipation components of SPDs, is made of mild steel or low-yield-point steel. Transverse and longitudinal units are reinforced all around to prevent the web from yielding too early. Web slenderness, $R_{w}$, is the key parameter affecting the hysteretic behavior and energy dissipation performance of an SPD and it can be defined and calculated according to Eq. 1, as proposed by Fukumoto [13]:

$$
R_{w}=\frac{b_{w}}{t_{w}} \sqrt{\frac{12\left(1-v^{2}\right) \tau_{y}}{k_{s} \pi^{2} E}}
$$

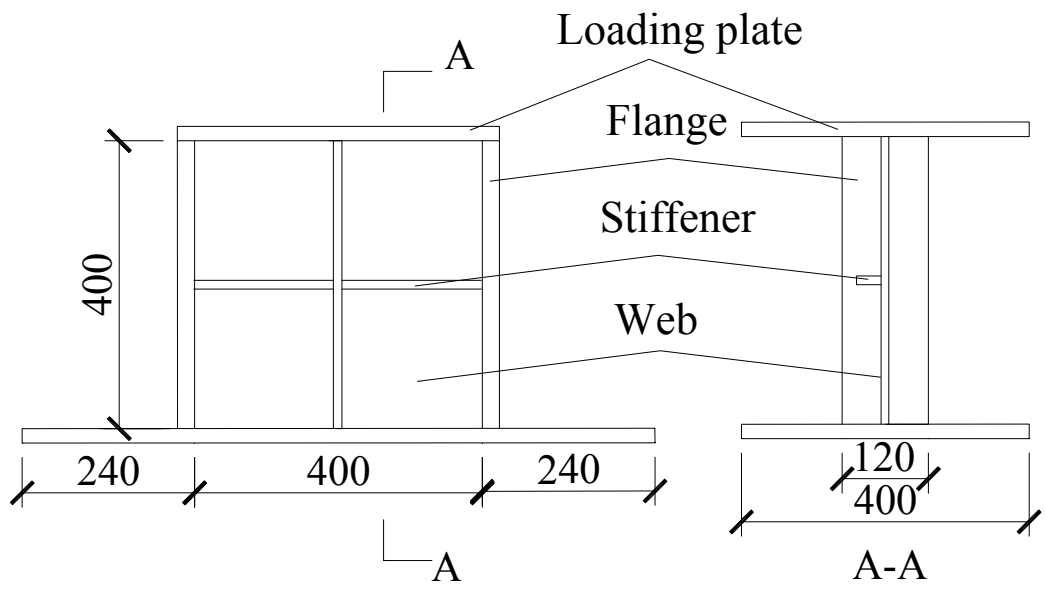

Figure 1. Schematic Diagram of Shear Panel Damper (Units: mm) 
Here, $b_{w}$ and $t_{w}$ are the width and thickness of the web, respectively, $v$ is Poisson's ratio, $\tau_{y}$ is the shear yield strength of the web material, $E$ is Young's modulus of elasticity, and $k_{s}$ is the elastic buckling coefficient of a simply supported plate:

$k_{s}= \begin{cases}\left(n_{L}+1\right)^{2}\left(5.35+1 / \alpha_{s}^{2}\right), & \alpha_{s} \geq 1 \\ \left(n_{L}+1\right)^{2}\left(5.35 / \alpha_{s}^{2}+4\right), & \alpha_{s}<1\end{cases}$

where $n_{L}$ is the number of longitudinal stiffeners and $\alpha_{s}$ is the aspect ratio of a subpanel.

Unstiffened SPDs have great ductility. However, they would yield in the early period of horizontal shear loading, leading to a reduction in shear strength and severe deterioration of their ability to dissipate energy. Therefore, a web plate is usually welded with a stiffener, effectively to prevent premature local buckling, to eliminate the pinch effect in the hysteresis loop, and to ensure a greater yielding range, thereby improving the energy dissipation capacity [14]. In this numerical analysis, the simulation model is stiffened in one-side and two-way (horizontal and vertical) modes. A two-way stiffener can efficiently prevent web buckling and degradation of the shear strength, allowing large deformation without conspicuous pinching of the hysteresis loop or reduction of the ultimate strength. Meanwhile, vertical stiffeners can resist part of the vertical axial compression force. Here, the width/thickness ratio is set at nine to avert premature local buckling. The stiffener rigidity is denoted as $\gamma_{s} / \gamma_{s} *$ [15], where $\gamma_{s}$ is the stiffness of the stiffener, which can be calculated using Eq. 3 and $\gamma_{s} *$ is the optimal stiffness of the stiffener, which can calculated using Eq. 4.

$$
\begin{aligned}
& \gamma_{s}=\frac{E I_{s}}{b_{w} D_{w}}, \\
& \gamma_{s}^{*}=\left(\frac{23.1}{n_{L}^{2.5}}-\frac{1.35}{n_{L}^{0.5}}\right) \frac{\left(1+\alpha^{3 / n_{L}-0.3}\right)^{2 n_{L}-1}}{1+\alpha^{5.3-0.6 n_{L}-3 / n_{L}}},
\end{aligned}
$$

where $I_{s}$ is the inertia moment, $D_{w}$ is the flexural rigidity per unit web width, and $\alpha$ is the aspect ratio of the full panel. It should be noted that the aspect ratio of the full panel is equal to that of the subpanel because the stiffeners are placed in equidistant pairs. The formulas are valid only when web parameters fall into the range of $n_{L}=n_{T} \leq 3$ and $0.5 \leq \alpha \leq 2.0$.

According to previous analysis results [16], $R_{w}$ is set as $0.2,0.3$, and 0.4 . In addition, an axial compression ratio, $z$, is defined as the ratio of the applied external axial force divided by the axial yielding force of the SPD. It is adopted to explore the effect of the axial compression force on the hysteretic behavior and energy dissipation performance of the SPDs. To clarify the effects of different magnitudes of the axial compression ratio, $z$ is taken as 0.0 (without axial compression), 0.3 (low axial compression), 0.5 (medium axial compression), and 0.7 (high axial compression). As stated in a prior parametric study [16], when the stiffener rigidity $\gamma_{s} / \gamma_{s}^{*}$, flange rigidity $t_{f} / t_{w}$, and web aspect ratio $\alpha$ satisfy: $\gamma_{s} / \gamma_{s} * \geq 3.0, t_{f} / t_{w} \geq 4.0$, and $0.5 \leq \alpha \leq 1.5$, the effects of these design parameters on the web shear strength can be neglected. Hence, the parameters in this paper are chosen as $\gamma_{s} / \gamma_{s}^{*}=3.0, t_{f} / t_{w}=4.0$, and $\alpha=1.0$. Numerical simulation parameters for SPDs are summarized in Table 1. 
Table 1. Summary of simulation parameters for SPDs

\begin{tabular}{cccccccccc}
\hline $\mathrm{z}$ & $R_{w}$ & $\begin{array}{c}b_{w} \\
(\mathrm{~mm})\end{array}$ & $\begin{array}{c}t_{w} \\
(\mathrm{~mm})\end{array}$ & $\begin{array}{c}t_{f} \\
(\mathrm{~mm})\end{array}$ & $\begin{array}{c}b_{f} \\
(\mathrm{~mm})\end{array}$ & $\begin{array}{c}t_{s} \\
(\mathrm{~mm})\end{array}$ & $\begin{array}{c}b_{s} \\
(\mathrm{~mm})\end{array}$ & $\begin{array}{c}P \\
(\mathrm{kN})\end{array}$ & $\tau_{m} / \tau_{y}$ \\
\hline 0.7 & 0.4 & 400 & 4.42 & 17.67 & 120 & 5.4 & 48.6 & 1031.6 & 1.159 \\
0.7 & 0.3 & 400 & 5.89 & 23.57 & 120 & 5.4 & 48.6 & 1361.3 & 1.303 \\
0.7 & 0.2 & 400 & 8.84 & 35.35 & 120 & 5.4 & 48.6 & 2020.5 & 1.445 \\
0.5 & 0.4 & 400 & 4.42 & 17.67 & 120 & 5.4 & 48.6 & 736.9 & 1.223 \\
0.5 & 0.3 & 400 & 5.89 & 23.57 & 120 & 5.4 & 48.6 & 972.3 & 1.365 \\
0.5 & 0.2 & 400 & 8.84 & 35.35 & 120 & 5.4 & 48.6 & 1443.2 & 1.654 \\
0.3 & 0.4 & 400 & 4.42 & 17.67 & 120 & 5.4 & 48.6 & 442.1 & 1.275 \\
0.3 & 0.3 & 400 & 5.89 & 23.57 & 120 & 5.4 & 48.6 & 583.4 & 1.448 \\
0.3 & 0.2 & 400 & 8.84 & 35.35 & 120 & 5.4 & 48.6 & 865.9 & 1.684 \\
0.0 & 0.4 & 400 & 4.42 & 17.67 & 120 & 5.4 & 48.6 & 0 & 1.395 \\
0.0 & 0.3 & 400 & 5.89 & 23.57 & 120 & 5.4 & 48.6 & 0 & 1.517 \\
0.0 & 0.2 & 400 & 8.84 & 35.35 & 120 & 5.4 & 48.6 & 0 & 1.666 \\
\hline
\end{tabular}

Note: $z$ : axial compression ratio; $R_{w}$ : web slenderness parameter; $b_{w}$ : width of web; $t_{w}$ : thickness of web; $b_{f}$. width of flange; $t_{f}$. thickness of flange; $b_{s}$ : width of stiffener; $t_{s}$ : thickness of stiffener; $P$ : axial force; $\tau_{m} / \tau_{y}$ : dimensionless maximum shear strenth; $\tau_{m}$ : maximum shear stress; $\tau_{y}$ : yield shear stress.

\subsection{Shear Angle Limit}

As a device that dissipates seismic energy through metal yielding, the SPD has an energy dissipation capacity that depends mainly on its plastic deformation capacity and fatigue performance. That is, an SPD must have sufficient ductility to withstand the maximum shear deformation suffered during an earthquake [16]. Here, the allowable maximum shear deformation is usually measured using a dimensionless parameter, namely the shear angle limit. Research conducted by Takahashi and Shinabe [17] suggests that when $R_{w}$ is less than 0.55 , the maximum shear angles of low-yield-point SPDs under a cyclic loading condition and monotonic loading condition are 0.05 and 0.1, respectively. Experiments carried out by Chen and Kuo [3] demonstrated that despite the shear angle reaching 0.06, the shear bearing capacity remained stable. The Code for Seismic Design of Subway Structures in Shanghai [18] specifies that the inter-story elastic-plastic drift angle limit for concrete-framed underground structures is $1 / 250$. Prior to the failure of SPDs, the vertical displacement of the flanges is relatively small. The shear deformation angle of the web, $\gamma$, can then be determined approximately according to the horizontal displacement divided by the height of the specimen $(0.4 \mathrm{~m})$. Considering that the two-yield-surface constitutive model used in numerical simulations is unable to simulate fatigue behavior [19], the maximum cyclic loading limit is set at 0.034 in the numerical analysis; i.e., the cyclic loading is terminated once the shear angle reaches 0.034 . For ordinary mild steel, this value is equivalent to $20 \gamma_{y}$ (where $\gamma_{y}$ refers to the yield shear strain, $\left.=0.002\right)$. Commonly, it is believed that within this range, the fatigue failure of steel under low-frequency cyclic action can be avoided; therefore, there is no necessity of modeling fracture in the numerical simulation. From the above discussion, it is safely concluded that the upper limit of an SPD shear angle is 0.034 . This value provides a safety and security margin in engineering practice. 


\subsection{Model Construction}

Figure 2 illustrates the numerical model of an SPD. It is clear that the shell elements S4 and S4R, which are provided by the ABAQUS element library, are available in this simulation. S4 is a four-node fully integrated, finite-membrane-strain shell element. The element's membrane response is treated with an assumed strain formulation that gives accurate solutions to in-plane bending problems. S4R is a four-node doubly curved, first-order, reduced integration shell element. S4 is compatible with S4R. However, S4 has four integration locations per element compared with one integration location for S4R, which makes S4 computationally more expensive. Considering that an SPD is subjected mainly to shear and axial forces, and that the two-yield-surface model used to simulate the material in the plastic phase is extremely computationally time-consuming, S4R is selected for the finite element modeling. The initial deformation and residual stress are considered. Depending on experimental inspections and the analytical study of plate assemblies [20], the distribution of the residual stresses in the web and the stiffeners is idealized in a rectangular pattern, as shown in Figure 3. Initial out-of-plane deflections have a sinusoidal distribution with the stiffener being the node of the curve and the amplitude being 1/150 of the height of the shear panel. To simulate accurately the large nonlinear deformation behavior of shear panels under cyclic loading, numerical simulations are based on a secondary-developed user subroutine platform provided by $A B A Q U S$, using the modified two-yield-surface model exploited by a research group at Nagoya University in Japan [21]. This model comprises a yield surface (the inner surface) and boundary surface (the outside surface) to describe the constitutive law of steel under cyclic loading. The yield surface is embodied permanently by the boundary surface. Additionally, the dimensions and correlation of these two surfaces determine the plastic modulus of the SPD. The modified two-yield-surface model is validated by the works of Gao et al. [22] and Chusilp et al. [23, 24], in which experiments were conducted on box girders to validate the modeling of shear behavior of mild steel under cyclic loading. The results showed that the modified two-yield-surface model performs well in tracing the material's nonlinearity under cyclic loading.

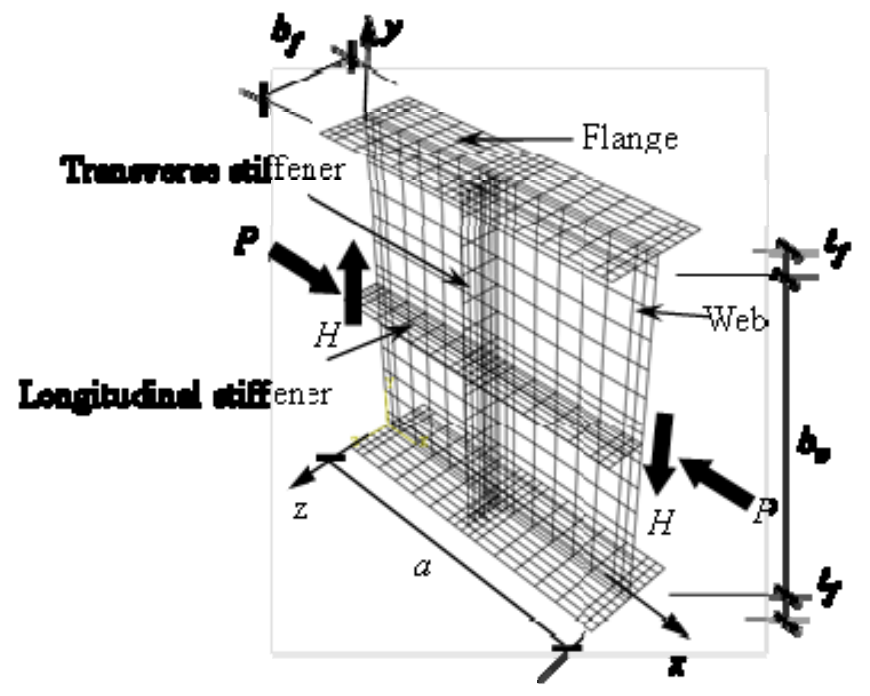

Figure 2. Finite Element Model 


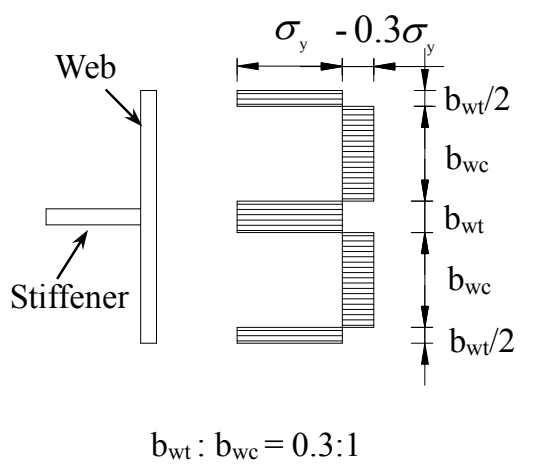

(a) Web

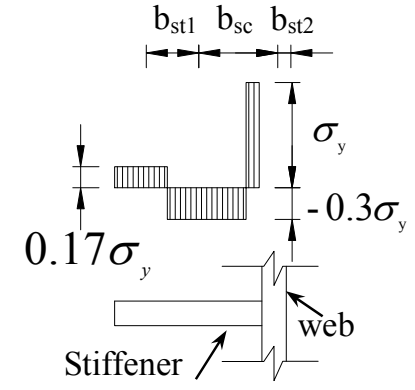

$b_{\mathrm{st} 1}: b_{\mathrm{sc}}: b_{\mathrm{st} 2}=0.69: 1: 0.17$

(b) Stiffener

Figure 3. Distribution of Residual Stresses

Taking into account the practical application, both ends of the panel are welded to a considerably stiff plate. Therefore, both ends of the model, at the planes $x=0$ and $x=a$ in Figure 2, are assumed to be fixed, except for the displacement in the $x$ and $y$ directions of the loading edge. Cyclic transverse displacement is applied to the shear panels along the plane $x=a$, and the displacement in each cycle $\Delta$ is the multiple of the yield displacement of the web, $\Delta_{y}$, in pure shear. The cyclic loading mode is illustrated in Figure 4. Then, the sum of the vertical reaction force in shear, $H$, is obtained along the plane $x=0$ to calculate the average shear stress, $\tau_{n}$, as shown in Eq. 5. In addition to the displacement loading, the SPD is also subjected to an axial compression $P$. As the axial compression is always translated to an SPD through a stiff loading plate, $P$ is uniformly distributed along the plane $x=a$ in the numerical analyses. The main results obtained from the numerical analyses are the average shear stress, $\tau_{n}$, and the average shear strain, $\gamma$, which are described by:

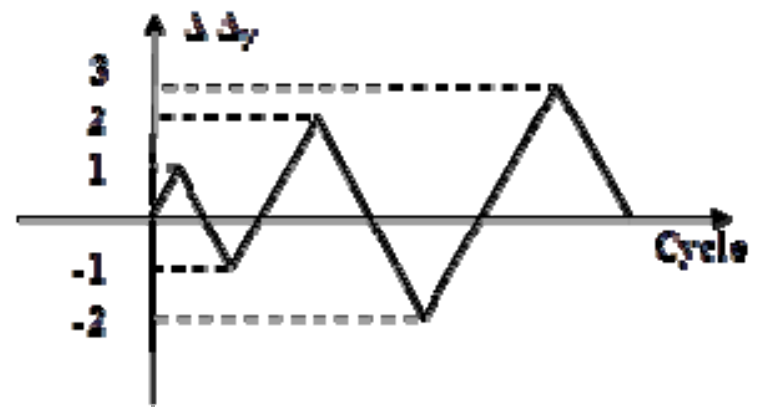

Figure 4. Loading Mode

$\tau_{n}=\frac{H}{b_{w} t_{w}} \quad \gamma=\frac{\Delta}{a}$

where $H$ is the sum of the vertical force in the plane $x=0$, and $\Delta$ is the vertical load displacement. 


\section{VERIFICATION AND VALIDATION OF THE NUMERICAL ANALYSIS MODEL}

This section aims to verify and validate the numerical model and simulation method using existing laboratory experimental results [5]. The specimen number is $R_{w} 03-z 07 . R_{w} 03-\mathrm{z} 07$ means that the tested specimen's web slenderness $R_{w}$ is 0.3 and the axial compression ratio $\mathrm{z}$ is 0.7 . That is, the axial load on the specimen is 0.7 times that of the axial yield strength of the cross-section. The dimensions of the shear-web specimen (see Figure 1) are 400 (width) $\times 400$ (height) $\times 6 \mathrm{~mm}$ (thickness). The vertical flange thickness is $24 \mathrm{~mm}$ and the width is $120 \mathrm{~mm}$. The areal dimensions of the loading plates are $450 \times 400 \mathrm{~mm}$ (upper) and $880 \times 400 \mathrm{~mm}$ (lower). The thicknesses of the loading plates are $20 \mathrm{~mm}$. Stiffeners are $40-\mathrm{mm}$ wide and 6-mm thick.

Figure 5 shows good agreement for (a) hysteretic curves and (b) cumulative dissipating energy between the laboratory test $\left(R_{w} 03-\mathrm{z} 07\right)$ and numerical simulation. The peak value of the dimensionless maximum shear strength, $H / H_{\mathrm{y}}$, is $1.369(\gamma=0.0162)$ in the numerical simulation and $1.378(\gamma=0.0145)$ in the laboratory test, as shown in Figure 5(a). The error is within $0.65 \%$. Additionally, the error in the normalized total cumulative dissipating energy is less than $0.82 \%$, as shown in Figure 5(b); this error is extremely low. The above comparisons demonstrate that the presented SPD model and numerical simulation method are valid. The hysteretic behavior and energy dissipation performance of SPDs under high axial compression can be predicted reliably in this manner.

\section{RESULTS AND DISCUSSION}

\subsection{Hysteretic Behavior}

Figure 6 shows hysteresis curves of SPDs with various values of $R_{w}$ and $z$. It can be seen that the strain-hardening phenomenon changes from isotropic hardening to a mix of kinematic and isotropic hardening with an increase in the axial compression ratio. Given a large value of $z$, the shear stress versus shear strain curves have obvious development stages, which include an elastic region, a yield region, a hardening zone, and a strength reduction stage. In the cases of $z=0.0$ and 0.3 , and $R_{w}=0.2$ and 0.3 , the hysteresis curves have a full shape and show the combined effects of kinematic and isotropic hardening. No web-buckling phenomenon is observed during the loading phase. Plastic deformation develops gradually throughout the entire steel web plate after yielding. Strain hardening occurs at $4 \gamma_{y}$ and remains until the end of the loading process.

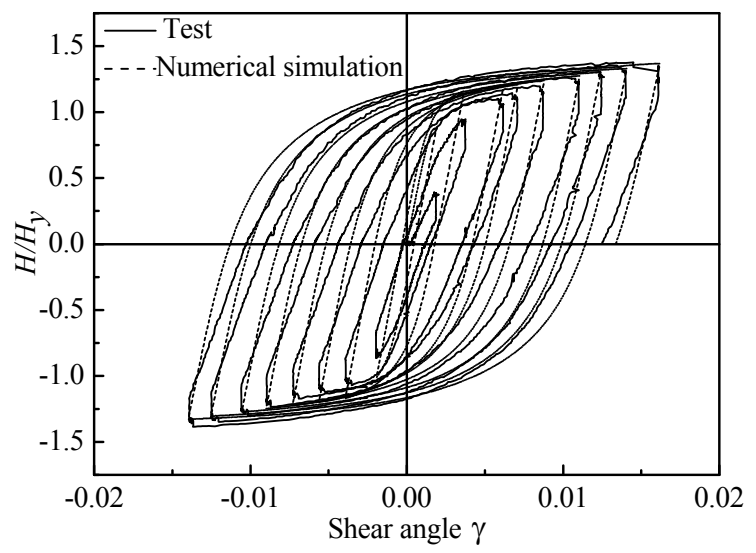

(a) Hysteretic Curves 


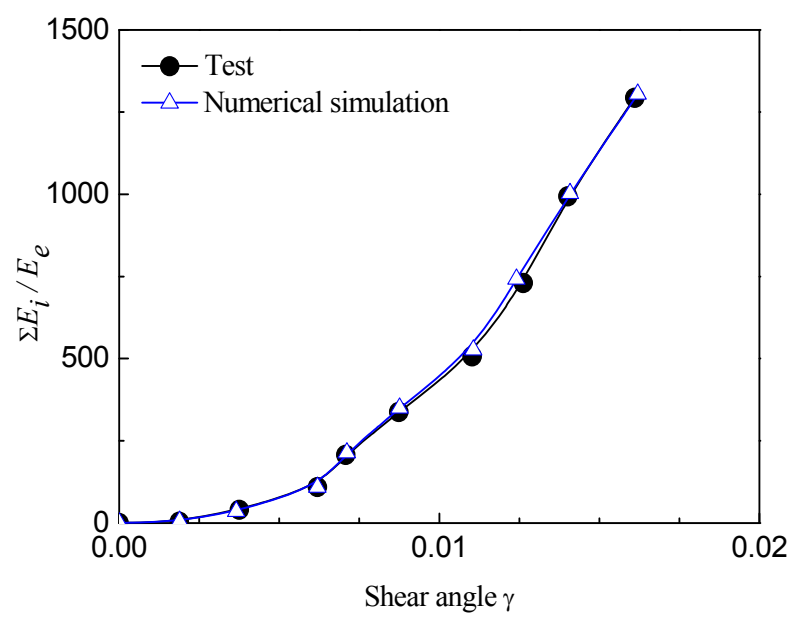

(b) Cumulative Energy Dissipation

Figure 5. Indoor Test versus Numerical Simulation of Specimen $R_{w} 03-z 07$

In the cases of $z=0.0,0.3$, and 0.5 , and $R_{w}=0.4$, the hysteresis curves are stable. Slight pinching, mainly due to web buckling, may be observed. However, pinching does not continue to develop under subsequent loading. Additionally, the average shear strength rises steadily until the end of loading.

In the cases of $z=0.5$, and $R_{w}=0.2$ and 0.3 , hysteresis curves are well developed and no pinching phenomenon occurs. However, several unstable hysteretic loops can be seen clearly at the end of loading. This is because the axial compression ratio is relatively high, which leads to unstable numerical computation results.

Under high axial compression $(z=0.7)$, hysteresis curves show significant strength degradation and even abrupt failure. Herein, high axial compression has become the dominant factor controlling the hysteretic performance of SPDs. It is found that the maximum shear deformation angle is about $9 \gamma_{y}$; hereafter, apparent out-of-plane deflection reduces the shear strength rapidly, and consequently, the calculation did not converge and the loading system was terminated.

It is concluded from the prior loading process that when the axial load is relatively small $(z=0.0$ or 0.3 ), its effect on the SPD under low cyclic loading action is rather limited. When the axial compression ratio is a mid-range value such as $z=0.5$, the specimen (such as in the case of $R_{w}=0.2$ ) has a hysteresis curve with evident fluctuations. This implies that relatively high axial compression has a certain effect on the shear bearing capacity of the SPD, which makes the SPD unstable. When the axial compression ratio is $z=0.7$, the hysteresis curve changes from a full shape to a curve with an obvious pinch. The result is a deterioration of the shear strength and even termination of the loading process. Greatly reduced energy dissipation is strong evidence of the detrimental effect of high axial compression on the hysteretic behavior of SPDs. Therefore, in practical applications, the compression ratio should be limited within a rational range. In design and construction, a large axial force on the dampers should be avoided. 


\subsection{Maximum Shear Strength}

The values of normalized maximum shear strength, $\tau_{m} / \tau_{y}$, are listed in Table 1 . The table shows that, given the same axial compression ratio, the maximum shear strength increases with a decrease in the web slenderness (or an increase in the web thickness). This is because not only the web but also the flanges contribute to the total shear strength. The thicker the web, the larger the resisting areas of the flanges, and the larger the contribution to the total shear. However, $\tau_{m}$ is the average shear divided only by the area of the web. On the other hand, given the same web slenderness, peak values of shear strength decrease with an increasing axial compression ratio. This can be observed more directly from the envelope of the shear stress versus shear strain shown in Figure 7.

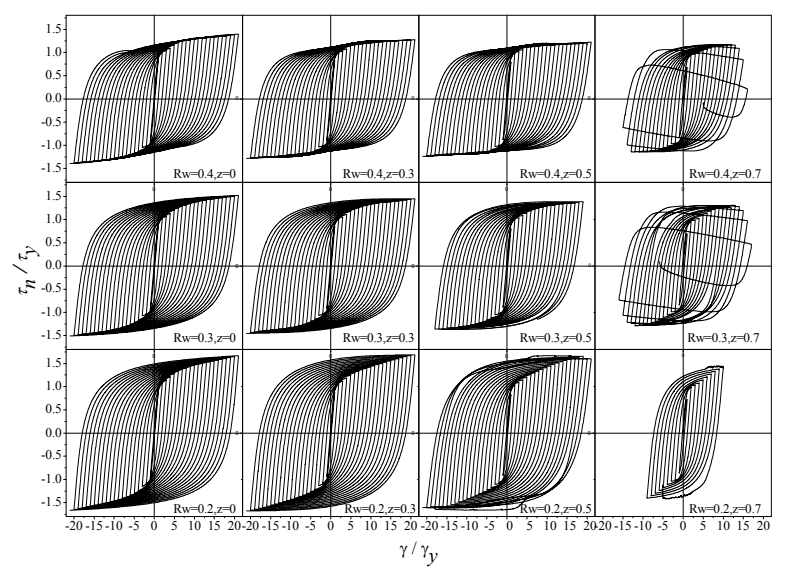

Figure 6. Shear Stress versus Shear Strain Hysteresis Curves

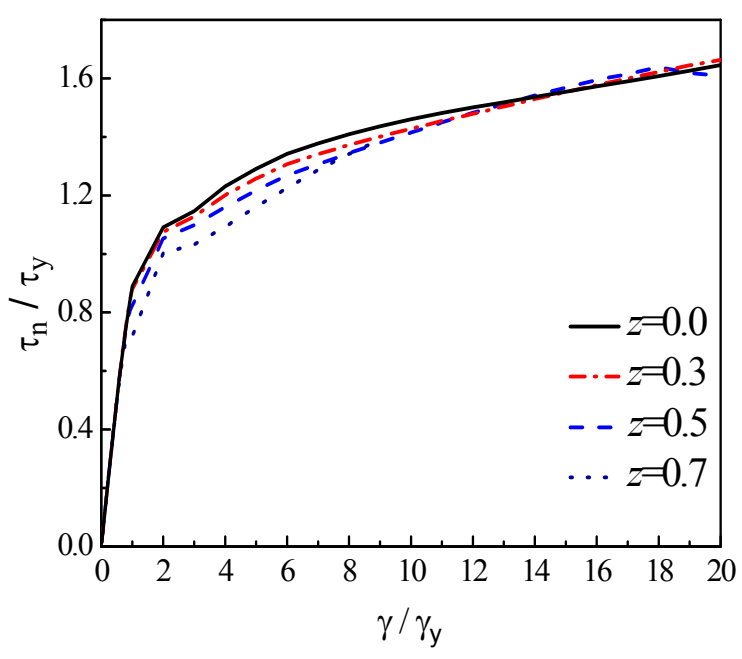

(a) $R_{w}=0.2$ 


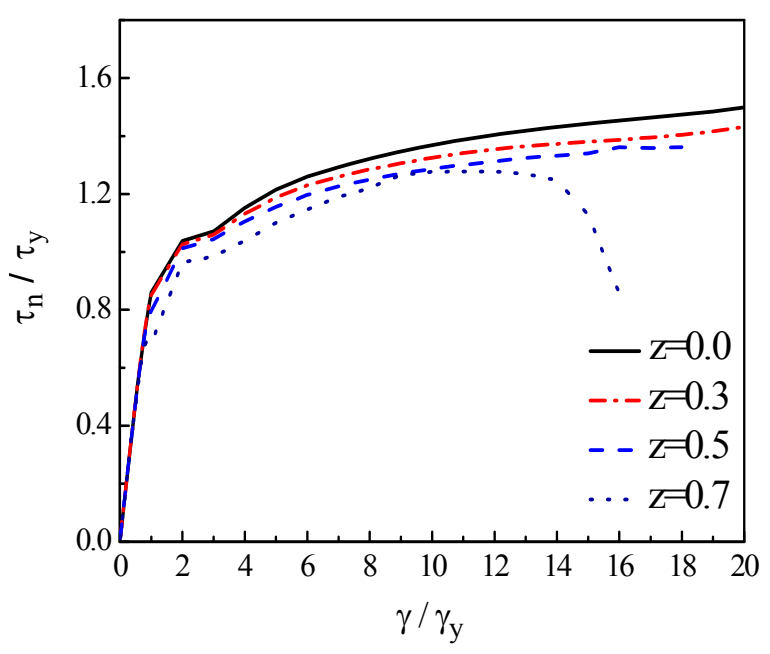

(b) $R_{w}=0.3$

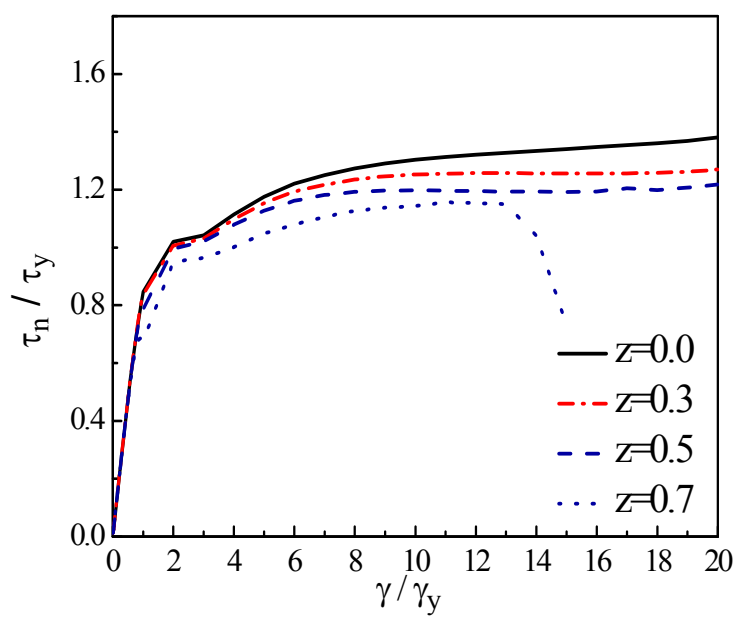

(c) $R_{w}=0.4$

Figure 7. Shear Stress versus Shear Strain Envelopes

Figure 7 shows skeletons of the hysteretic curves presented in Figure 6. The envelope curve is drawn by connecting the mean values of absolute values of shear stress that correspond to the maximum shear strain in each loading and unloading cycle. When the value of $R_{w}$ falls within the range of $0.2-0.4$, SPDs are prone to enter the plastic stage earlier with an increase in the axial compression ratio. In the case of $R_{w}=0.2$, an increase in the axial compression ratio reduces the shear bearing capacity only in the initial stage of plastic deformation. In the subsequent large-deformation stage, the envelopes of the shear stress versus shear strain of the specimens are almost identical, except for the case of $z=0.7$, in which the loading process is terminated suddenly. In the cases of $R_{w}=0.3$ and 0.4 , however, the increase of the axial compression ratio is accompanied by a gradual degradation of the shear bearing capacity.

When $R_{w}$ varies in the range of $0.2-0.4$ and the axial compression ratios are comparatively preferable (i.e., $z=0.0,0.3$, and 0.5 ), the skeleton curves ascend slowly as the shear deformation angle increases because of the strain hardening of mild steel. Shear bearing capacities of SPDs remain stable without deterioration. The shear bearing capacity at the moment loading ceases transpires as the maximum shear strength in the loading test. Meanwhile, under a high axial 
compression ratio when $z=0.7$ and $R_{w}=0.3$ and 0.4 , the maximum shear strength is $1.246 \tau_{y}$ and $1.149 \tau_{y}$, respectively, and the corresponding shear deformation angle is $14 \gamma_{y}$ and $13 \gamma_{y}$, respectively. There is a subsequent dramatic decrease in the shear bearing capacity and the situation continues until the specimens are destroyed. In the case of the specimen with a thicker web under the same high axial load (i.e., $z=0.7, R_{w}=0.2$ ), the envelope ceases unexpectedly at $9 \gamma_{y}$ and the damper is damaged through brittle failure, similar to the case of concrete members. Previous laboratory experiments have revealed that the failure of the SPD specimens is primarily due to material fracture or large deformation of out-of-plain buckling. As stated before, fatigue failure (fracture) of steel material will not appear and may be ignored in simulations given the imposed shear deformation within $20 \gamma$. Therefore, it could be concluded reasonably that the difficulty in convergence is due to out-of-plain buckling, which leads to the brittle failure of the specimen. Previous research has shown that under no axial compression and with the same web width condition, the lower the slenderness, the thicker the web and consequently, the larger the corresponding shear bearing capacity. However, under high axial compression, the hysteretic performances of SPDs deteriorate remarkably, as characterized by the brittle failure, apparently due to the combined hazards of the shear force and axial compression. Thus, low slenderness is not conducive to improving the bearing capacity and energy dissipation performance of SPDs when there is high axial compression.

\subsection{Energy Dissipation Performance}

Energy dissipation performance is one of the most important indicators for evaluating an SPD as an energy dissipation device. Dissipated energy for each hysteresis circle, $E_{i}$, is calculated in the manner illustrated in Figure 8. The cumulative dissipation energy $\sum E_{i}$ is then calculated and normalized by $E_{e}$ (here $E_{e}=H_{y} \Delta_{y} / 2$ ) to give the relationship versus cycle number, as shown in Figure 9.

When $z=0.0$ and $0.3, \sum E_{i} / E_{e}$ increases with a decrease in $R_{w}$. This is mainly because the area of metal, which goes into a plastic state, increases. When $z=0.5$ or 0.7 , there is no significant relation between the cumulative dissipation energy and the web slenderness. In fact, the high axial compression becomes the dominant factor controlling the energy dissipation performance of the SPDs.

On the other hand, from the aspect of the axial compression ratio, when $R_{w}$ is comparatively large (i.e., $R_{w}=0.4$ or 0.3 ), the cumulative dissipation energy drops slightly in the wake of an increasing axial compression ratio. When web slenderness $R_{w}$ is relatively small (i.e., $R_{w}=0.2$ ), the cumulative dissipation energy is amplified a little as the axial compression ratio increases from 0 to 0.5 . This can be explained by the increasing web thickness, which makes it possible for a greater cross-section of metallic materials to enter the plastic energy dissipation stage. That is, enlarging the cross-section of an SPD to some extent benefits the resistance to the imposed axial compression. In general, SPDs have good shear bearing capacity and energy dissipation capacity when the axial compression ratio is less than or equal to 0.5 . What should be noted, however, is that the hysteretic behavior degrades severely as the axial compression ratio reaches 0.7 . Under this circumstance, the SPD goes into an unstable state and fails abruptly, even if the shear deformation is small. This leads to the dramatic deterioration of cumulative energy dissipation. 


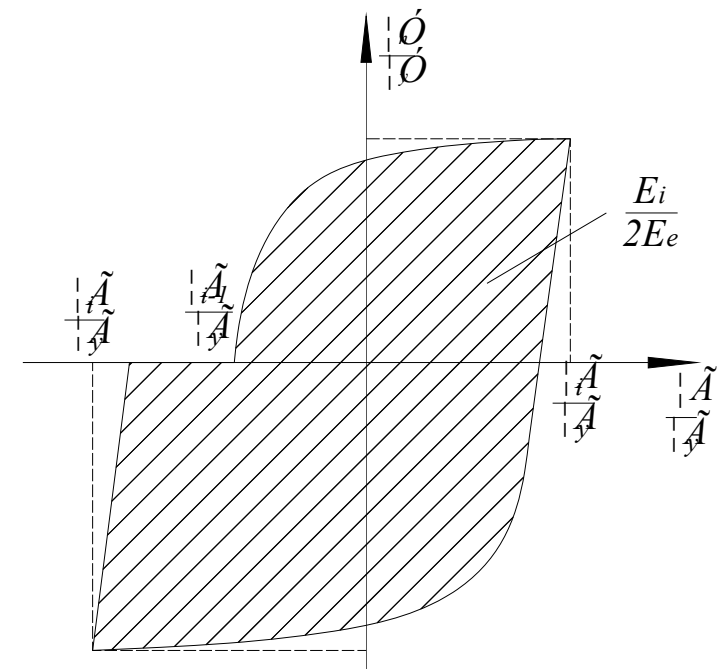

Figure 8. Schematic Diagram of Accumulated Energy

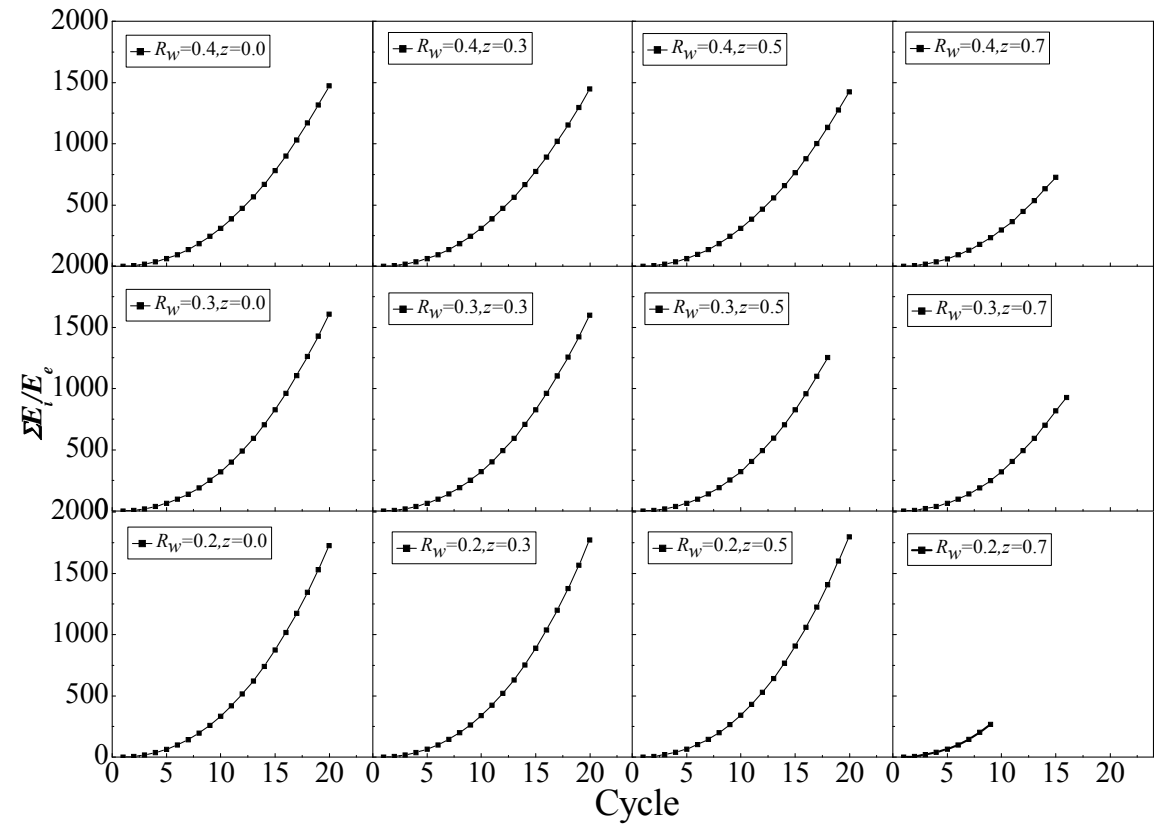

Figure 9. Cumulative Energy Dissipation

\section{CONCLUSIONS}

Extensive numerical analyses were conducted for stiffened SPDs under axial compression. The effects of the axial compression ratio and the web slenderness parameter on hysteretic behavior and energy dissipation performance were explored. The main findings were as follows:

1) Axial compression deteriorates the hysteretic behavior and energy dissipation performance of SPDs. Under relatively small axial compression $(z<0.5)$, negative impacts are negligible, whereas under high axial compression $(z=0.7)$, the ductility, shear bearing capacity, hysteresis, and energy dissipation of SPDs deteriorate remarkably. 
2) The web slenderness parameter is a governing parameter for SPDs. Generally, a decrease in web slenderness benefits the shear strength and cumulative energy dissipation capacity under low and medium axial compression ratios, similar to cases without axial compression. However, in the case of high axial compression ratio, a small web slenderness parameter will lead to unexpected brittle failure (in the case of $z=0.7, R_{w}=0.2$ ). This is undesirable in the design of SPDs.

\section{ACKNOWLEDGMENTS}

This research was supported by the National Natural Science Foundation of China (Grant No. 51278524), the Program for Changjiang Scholars and Innovative Research Team in University (PCSIRT, IRT1029), and the State Key Laboratory of Disaster Reduction in Civil Engineering (SLDRCE12-MB-02). All supports are acknowledged gratefully.

\section{REFERENCES}

[1] Nakashima, M., "Strain-hardening Behavior of Shear Panels Made of Low-yield Steel.1: Test", Journal of Structural Engineering, 1995, Vol. 121, No. 12, pp. 1742-1749.

[2] Nakashima, M., Akazawa, T. and Tsuji, B., "Strain-hardening Behavior of Shear Panels Made of Low-yield Steel.2: Model", Journal of Structural Engineering, 1995, Vol. 121, No. 12, pp. 1750-1757.

[3] Chen, S.J. and Kuo, C.L., "Experimental Study of Vierendeel Frames With LYP Steel Shear Panels Steel Structures", Steel Structures, 2004, Vol. 4, pp. 179-186.

[4] Chen, Z.Y., Ge, H.B. and Usami T., "Study on Seismic Performance Upgrading for Steel Bridge Structures by Introducing Energy-dissipation Members", Journal of Structural Engineering, JSCE, 2007, Vol. 53, pp. 540-549.

[5] Chen, Z.Y., Bian, G.Q. and Huang, Y., "Hysteretic Behavior of Shear Panel Dampers under High Axial Compression Loading”, Advanced Steel Construction, 2013, Vol. 9, No. 3, pp. 185-199.

[6] Ohta, Y., Kaneko, H., Kibayashi, M., Yamamoto, M., Muroya, T. and Nakane, K., "Study On Shear Panel Dampers Using Low Yield Strength Steel Applied To Reinforced Concrete Buildings", 13th World Conference on Earthquake Engineering, Vancouver, B.C., Canada, 2004, No. 2228.

[7] Galal, K.E. and Ghobarah, A., "Flexural and Shear Hysteretic Behavior of Reinforced Concrete Columns with Variable Axial Load", Engineering Structures, 2003, Vol. 25, No. 11, pp. 1353-1367.

[8] Gustavo, J.P.M., Bobet, A. and Ramirez, J., "Evaluation of Soil-structure Interaction and Structural Collapse in Daikai Subway Station during Kobe Earthquake", ACI Structural Journal, 2006, Vol. 103, No. 1, pp. 113-122.

[9] Huo, H., Bobet, A., Fernández, G. and Ramírez, J., "Load Transfer Mechanisms between Underground Structure and Surrounding Ground: Evaluation of the Failure of the Daikai Station", Journal of Geotechnical and Geoenvironmental Engineering, 2005, Vol. 131, No. 12, pp. 1522-1533.

[10] Sasaki, Y. and Tanaka, K., "Static Hysteretic Performance of Shear Panel Damper with Low Yield Strength Steel under Constant Axial loads", Journal of Fujita Technical Research Institute, 1999, No. 35, pp. 61-66.

[11] Nakashima, M., Iwai, S., Iwata, M., Takeuchi, T., Konomi, S., Akazawa, T. and Saburi, K., "Energy Dissipation Behavior of Shear Panels Made of Low Yield Steel", Earthquake Engineering and Structural Dynamics, 1994, Vol. 23, No. 12, pp. 1299-1313. 
[12] GB/T 19879, "Steel Plates for Building Structure”, the People's Republic of China State Administration of Quality Supervision, Inspection and Quarantine \& China National Standardization Management Committee, Beijing: Standards Press of China, 2005.(in Chinese)

[13] Fukumoto, Y. ed., "Guidelines for Stability Design of Steel Structures", Subcommittee on Stability Design, Committee on Steel Structures, Japan Society of Civil Engineers, Tokyo, Japan, 1987. (in Japanese)

[14] Alinia, M.M., Dastfan, M., "Cyclic Behavior, Deformability and Rigidity of Stiffened Steel Shear Panels", Journal of Constructional Steel Research, 2007, Vol 63, No. 4, pp. 554-563.

[15] Chusilp, P. and Usami, T., "New Elastic Stability Formulas for Multiple-stiffened Shear Panels", Journal of Structural Engineering, ASCE, 2002, Vol. 128, No. 6, pp. 833-836.

[16] Chen, Z.Y., Ge, H.B., Kasai, A. and Usami, T., "Numerical Study on Development of Hysteretic Model for Stiffened Steel Shear Panel Dampers", Journal of Structural Engineering, JSCE, 2006, Vol. 52, pp. 573-582.

[17] Takahashi, Y. and Shinabe,Y., "Experimental Study on Restoring Force Characteristics of Shear Yielding Thin Steel Plate Elements", Journal of Structural Engineering, AIJ, 1997, Vol. 494, pp. 107-114.

[18] DG/TJ08-2064-2009, "Code for Seismic Design of Subway Structures”, Shanghai Urban Constructions Communications, Shanghai, 2010. (in Chinese)

[19] Kasai, A., Watanabe, T., Amano, M. and Usami, T., "Strength and Ductility Evaluation of Stiffened Steel Plates Subjected to Cyclic Shear Loading”, Journal of Structural Engineering, 2001, Vol. 47, No.2, pp. 761-770.

[20] Usami T., Ge, H.B., "Strength Prediction of Thin-walled Plate Assemblies", Journal of Structural Engineering, JSCE, 1996, Vol. 42, pp. 171-178.

[21] Shen, C., Mamaghani, I.H.P, Mizuno, E. and Usami, T., "Cyclic Behavior of Structural Steels. II: Theory”, Journal of Engineering Mechanics, 1995, Vol. 121, No. 11, pp. 1165-1172.

[22] Gao, S.B., Usami, T., and Ge, H.B., "Ductility evaluation of steel bridge piers with pipe sections", Journal of Engineering Mechanics, ASCE, 1998, Vol. 124, No. 3, pp.260-267.

[23] Chusilp, P., Usami, T., Ge, H.B., Maeno, H., and Aoki, T., "Cyclic shear behavior of steel box girders: experiment and analysis", Earthquake Engineering \& Structural Dynamics, 2002, Vol. 31, No. 11, pp.1993-2014.

[24] Chusilp, P., "Seismic Design Methodology for Steel Bridge Structures with Shear-type Hysteric Dampers", Thesis, presented to Nagoya University, Japan, in partial fulfillment of the requirements for the degree of Doctor of Engineering, 2002. 